\title{
Correspondence
}

\section{Persistent perioperative laryngospasm in a patient with Parkinson's disease}

To the Editor:

A 61-yr-old man with Parkinson's disease (PD) had surgery for a perianal abscess. He was wheelchair bound, had impaired speech but no dyspnoea, upper airway obstruction or dysphagia. He had a receding chin, and limited jaw movement due to previous surgery. Treatment with Sinemet (levodopa and carbidopa), benzhexol and bromocriptine was continued throughout the perioperative period.

General anaesthesia was induced with propofol, and maintained with sevoflurane and fentanyl. As anaesthetic depth increased, he developed airway obstruction. An oropharyngeal airway was inserted. This and surgical stimulation caused laryngospasm, which lessened as anaesthetic depth was further increased. At laryngoscopy, only the epiglottis could be seen. The oropharynx was clear. Laryngospasm persisted and worsened after surgery and full awakening. Awake blind nasal intubation immediately relieved airway obstruction and he could then breathe well. We avoided muscle relaxants as conventional intubation would have been difficult. His chest was clear and he had no new neurological signs.

On extubation $24 \mathrm{hr}$ later, laryngospasm recurred. Fibreoptic laryngoscopy showed the vocal cords adducting during inspiration. There was no airway inflammation or lesion. As laryngospasm worsened, the trachea was re-intubated, Sinemet dosage was doubled and uneventful extubation was performed $24 \mathrm{hr}$ later.

Primary laryngospasm is a known complication of $\mathrm{PD}^{1,2}$ and acute withdrawal of treatment can cause airway obstruction. ${ }^{3}$ While laryngospasm in this patient was triggered by airway stimulation, persistent laryngospasm was due to inadequate treatment. While seemingly adequate treatment was not interrupted, an increased dosage was required to relieve laryngospasm. Parkinsonian patients may need optimization of their treatment during an acute infection and in the perioperative period to prevent persistent laryngospasm.

\footnotetext{
E.H. Liu, J. Choy, S.S. Dhara

Department of Anaesthesia

National University Hospital

Lower Kent Ridge Road

Singapore 119074
}

\section{REFERENCES}

1 Vincken WG, Gautbier SG, Dollfuss RE, Hanson RE, Darauay $C M$, Cosio $M G$. Involvement of upper airway muscles in extrapyramidal disorders. A cause of airflow limitation. N Engl J Med 1984; 311: 438-42.

2 Fikkers BF, Zandstra DF. Primary laryngospasm in a patient with Parkinson's disease: treatment with CPAP via a minitracheostomy following intubation. Intensive Care Med 1995; 21: 863-4.

3 Easdown LJ, Tessler MJ, Minuk J. Upper airway involvement in Parkinson's disease resulting in postoperative respiratory failure. Can J Anaesth $1995 ; 42: 344-7$.

\section{Another potential problem with the "bidden iv"}

\section{To the Editor:}

Surgeons often request one or both arms tucked beside the anaesthetized patient's body during surgery. The iv catheter, as it enters the vein, is therefore hidden from view. Serious complications can arise when the $i v$ insertion site is hidden. One of the more serious being the infiltration of fluids into the hand/arm. One of the authors (JGBU) has seen a case where the arm above the elbow had to be amputated following a five hour cardiac bypass procedure. We recently had another potential problem with the "hidden iv" which we believe is unreported.

A 55-yr-old man was scheduled for emergency laparatomy. $\mathrm{He}$ arrived in the operating room with a $20 \mathrm{G}$ iv catheter in his left hand. This was seen to be working adequately and general anaesthesia was induced in a routine manner using this line. After induction, a $14 \mathrm{G}$ peripheral iv and a central vascular line were inserted into the right subclavian vein without any problems. The left arm, with the $20 \mathrm{G}$ iv, was tucked alongside the patient's body. During the operation, renal dose dopamine was needed. We elected to use the central line instead of the $20 \mathrm{G}$ iv line which, incidentally, was seen to be working well. The urine output improved and the patient was taken to the recovery room, awake and cooperative with stable vital signs. Here we discovered that, $20 \mathrm{~cm}$ from the insertion of the $20 \mathrm{G} i \mathrm{v}$, the intravenous line was cut. The distal end, attached to the $20 \mathrm{G} i v$ catheter, had a tight knot while the proximal end was open. We now 